\title{
Etude ethnobotanique des plantes utilisées contre le paludisme par les tradithérapeutes et herboristes dans le district d'Abidjan (Côte d'Ivoire)
}

\author{
Youssouf SYLLA ${ }^{1}$, Dieudonné Kigbafori SILUE ${ }^{2,3}$, Kigninma OUATTARA ${ }^{2}$ et \\ Mamidou Witabouna KONE ${ }^{1,3 *}$
}

\author{
${ }^{1}$ UFR Sciences de la Nature, Université Nangui Abrogoua, BP 801 Abidjan 02, Côte d'Ivoire. \\ ${ }^{2}$ UFR Biosciences, Université Félix Houphouët-Boigny, 22 BP 582 Abidjan 22, Côte d'Ivoire. \\ ${ }^{3}$ Centre Suisse de Recherches Scientifiques en Côte d'Ivoire, 01 BP 1303 Abidjan 01, Côte Ivoire. \\ *Auteur correspondant; E-mail: konewit_sn@una.edu.ci; mamidou.kone@csrs.ci; Tel: + 22503488905.
}

\section{RESUME}

Le paludisme est une maladie endémique en Afrique subsaharienne. La prise en charge est contrariée par les résistances aux médicaments antipaludiques. Cette situation suscite un intérêt pour des alternatives thérapeutiques, y compris les plantes médicinales. L'objectif de cette étude était d'évaluer la diversité des plantes médicinales utilisées pour traiter le paludisme. Des enquêtes ethnobotaniques ont été menées auprès de 83 acteurs de la médecine traditionnelle choisis au hasard à l'aide d'entretiens semi-structurés dans le district d'Abidjan. Ces enquêtes ont permis d'inventorier 54 espèces de plantes appartenant à 29 familles. Les rubiacées et les combrétacées $(5$ espèces ; 9,25\%) suivies des Fabacées et des Méliacées (4 espèces ; 7,41\%) sont les familles les plus représentées. Les organes végétaux les plus utilisés dans les différentes recettes sont les feuilles $(68,89 \%)$ et la méthode de préparation la plus proposée par les tradithérapeutes est la décoction (76,97\%). La plupart des préparations sont administrées par voie orale $(84,09 \%)$ sous forme de boisson. Ces résultats pourraient constituer une base d'information pour la recherche de nouvelles molécules à potentiel antipaludique et au développement de médicaments traditionnels améliorés (MTA).

(C) 2018 International Formulae Group. All rights reserved.

Mots clés: Ethnobotanique, plantes médicinales, paludisme, tradithérapeutes, herboristes, Côte d'Ivoire.

\section{Ethnobotanical study of medicinal plants used by traditional healers and herbalists against malaria in the district of Abidjan (Côte d'Ivoire)}

\begin{abstract}
Malaria is an endemic disease in sub-Saharan Africa. The control of this disease is curb by the resistance to antimalarial drugs. This situation raises interest for therapeutic alternatives including medicinal plants. The objective of this study was to evaluate the diversity of medicinal plants used to treat malaria. Ethnobotanical surveys were carried out among 83 randomly selected herbalists using semi-structured interviews in the Abidjan district. A total of 54 species of plants belonging to 29 families, was censused. Rubiaceae and Combretaceae (5 species, 9.25\%) followed
\end{abstract}


by Fabaceae and Meliaceae (4 species, $7.41 \%$ ) were the most represented families. The plant organs mostly used in the different receipts were leaves $(68.89 \%)$ and concoction $(76,97 \%)$ were the most method used for preparing receipts. A great part $(84,09 \%)$ of these medicines were administered orally as a drink. These results may be a database for the discovery of new molecules with antimalarial potential and the development of improved traditional medicines (ITM).

(c) 2018 International Formulae Group. All rights reserved.

Keywords: Ethnobotany, medicinal plants, malaria, traditional healers, herbalists, Côte d'Ivoire.

\section{INTRODUCTION}

Depuis toujours et dans toutes les tribus à travers le monde, l'Homme se soigne par les plantes. Selon l'OMS (2004), $80 \%$ de la population mondiale se soigne encore plus ou moins complètement par les plantes, fautes de pouvoir accéder aux médicaments modernes, du fait de leur coût élevé. La médecine traditionnelle apparaît, donc, comme une alternative pour ces populations économiquement faibles surtout dans les pays d'Afrique subsaharienne où sévissent de nombreuses maladies infectieuses. Parmi ces pathologies, le paludisme reste de loin celle qui enregistre le taux de mortalité le plus important (OMS, 2011 ; Bla et al., 2015). Cette endémie des zones tropicale et subtropicale est due à un hématozoaire du genre Plasmodium qui est transmis par la piqûre d'un moustique, l'Anophèle femelle (Pradines et al., 2010). Le paludisme représente un problème majeur de santé publique avec près de 3,3 Milliards de personnes soit plus de la moitié de la population mondiale exposée au risque de cette maladie. L'Afrique est le continent le plus touché avec près de 300 à 500 millions de cas cliniques par an dont $90 \%$ en Afrique subsaharienne et une mortalité annuelle estimée entre 1,5 et 2,7 millions d'individus (OMS, 2016). Face à ce tableau sombre et surtout à l'émergence de résistance de souches de Plasmodium aux antipaludiques même aux plus récents tels que les combinaisons thérapeutiques à base d'artémisinine, un regain d'intérêt est accordé à la découverte de nouvelles molécules à partir de plantes issues des pharmacopées d'Afrique et d'Asie (Pradines et al., 2010). Depuis lors, en Côte d'Ivoire, ces dernières décennies ont été marquées par la réalisation de nombreux travaux consacrés, dans la plupart des cas à des enquêtes ethnobotaniques portant sur des plantes médicinales par des auteurs tels que Koné (2005), Zirihi (2006), Ouattara (2006), N'Guessan (2008), Bla et al. (2015) et Kipré et al. (2017). Dans cette lancée, une étude ethnobotanique dans le district d'Abidjan, carrefour par excellence de rencontre et d'échange entre tradithérapeutes et herboristes de différentes régions du pays et même de la sous-région, s'avère judicieuse pour contribuer aux investigations et répertorier de nouvelles plantes à potentialité antipaludique. C'est ainsi que cette étude ethnobotanique a été menée dans le district d'Abidjan avec pour objectif principal d'évaluer la diversité des plantes médicinales utilisées par les tradithérapeutes et herboristes pour traiter le paludisme.

\section{MATERIEL ET METHODES Zone d'étude}

Cette étude a été menée dans les 10 communes du district autonome d'Abidjan (Abobo, Adjamé, Attécoubé, Marcory, Treichville, Koumassi, Yopougon, PortBouët, Plateau, Cocody). Le district d'Abidjan est situé au Sud forestier, dans la région des Lagunes. Il s'étend sur une superficie de 2119 $\mathrm{Km}^{2}$ et ses coordonnées géographiques sont comprises entre $5^{\circ} 20^{\prime} 27^{\prime \prime}$ de latitude Nord et $4^{\circ} 01^{\prime} 41^{\prime \prime}$ de longitude Ouest (Figure 1). La population $\mathrm{du}$ district est fortement diversifiée, cosmopolite et composée de plusieurs peuples et groupes ethniques venant de diverses régions du pays mais aussi des pays voisins. Le climat est de type tropical et humide appelé localement "climat Attiéen » avec quatre saisons. On distingue une grande saison sèche (janvier-février), une grande saison des pluies (mars-juillet) avec le maximum de précipitation en juin, une petite saison sèche (août) et une petite saison des pluies (septembre-décembre) avec le maximum de précipitation en octobre 
(SODEXAM, 2015). La pluviométrie annuelle est comprise entre 1400 et $2400 \mathrm{~mm} / \mathrm{an}$ avec un taux d'humidité élevé. La végétation est de type forestier naguère dense mais fortement réduite par l'urbanisation.

\section{Méthode d'étude \\ Identification et choix des tradithérapeutes et herboristes}

Les tradithérapeutes et herboristes ont été identifiés et choisis de façon aléatoire dans chacune des dix communes de la ville d'Abidjan sur la base des données du Programme National de Promotion de la Médecine Traditionnelle (PNPMT). Des contacts ont été pris avec des tradithérapeutes et herboristes qui ont bien voulu participer à l'enquête. Des rendez-vous ont été généralement pris soit dans leur cabinet, soit à leurs domiciles ou dans les marchés sur les sites de ventes des plantes médicinales. Le Tableau 1 présente la répartition des enquêtés par commune.

\section{Enquêtes ethnobotaniques}

Les enquêtes ont été menées entre novembre 2014 et janvier 2015. Elles ont consisté en des interviews semi-structurées à partir d'un questionnaire. Ces entretiens ont porté sur le statut socio-démographique des tradithérapeutes et des herboristes, sur leur niveau de connaissance du paludisme, sur les plantes ou organes de plantes utilisées dans les différentes recettes ainsi que leurs modes de préparation et d'administration. Chaque entretien avec les tradithérapeutes était suivi de sorties de terrain en vue de récolter des échantillons des espèces citées ou d'en acheter auprès des herboristes et ce, sur la base des noms vernaculaires de ces plantes. Des herbiers ont été confectionnées et l'identification des espèces a été effectuée au Centre Suisse de Recherches Scientifiques en Côte d'Ivoire (CSRS) puis authentifiées par comparaison avec l'herbier du Centre National de Floristique (CNF) de 1'Université Felix Houphouët Boigny (UFHB). Pour harmoniser la classification des taxons, le système de nomenclature APG IV a été utilisé

\section{Traitement des données}

Après le dépouillement des fiches d'enquêtes, l'analyse des données a porté sur la détermination, pour chaque espèce, de la fréquence de citation (FC), de la contribution dans la constitution des recettes (CPr) et de l'indice de confirmation ou consensus d'informateurs (ICs).

\section{Fréquence de citation}

La fréquence de citation (FC) de chaque espèce permet d'apprécier la régularité dans la distribution d'une espèce. Elle a été déterminée, pour chaque espèce, par la formule :

\section{FC $=$ NP/NTx100}

Avec NP: nombre de fois où l'espèce est citée, NT : nombre total de citations

\section{Contribution de chaque espèce}

$\mathrm{La}$ Contribution de chaque espèce $(\mathrm{CPr})$ permet d'apprécier la fréquence d'implication de cette espèce dans les recettes. Elle a été déterminée pour chaque espèce par la formule :

$$
\mathrm{CPr}=(\mathrm{Nr} / \mathrm{Nt}) \times 100
$$

Avec $\mathrm{Nr}$ : nombre de recettes sollicitant la plante, Nt : nombre total de recettes.

\section{Indice de confirmation}

L'Indice de confirmation ou Consensus d'informateurs (ICs) permet d'apprécier les accords des informateurs sur les plantes utilisées. Il a été également calculé pour chaque espèce par la formule utilisée par Ilumbe et al. (2014) qui se présente comme suit :

$$
\mathrm{ICs}=\mathrm{Na} / \mathrm{Nt}
$$

Avec $\mathrm{Na}=$ nombre de personnes ayant cité cette espèce et $\mathrm{Nt}=$ nombre total de personnes interviewées. L'ICs varie entre 0 et 1 . Une valeur faible, proche de 0 , indique que les informateurs sont en désaccord sur les plantes utilisées. Une valeur élevée, proche de 1, indique un consensus élevé ou total autour de l'usage de la plante.

\section{Analyses statistiques}

Les données des enquêtes ont été saisies en utilisant le logiciel Epidata 3.1 puis transférées sur le logiciel SPSS 20.0 pour les analyses statistiques. Les tableaux et les figures ont été réalisés avec le logiciel SPSS 20.0 pour mieux apprécier et expliquer le niveau de connaissance du paludisme, les plantes utilisées pour le traiter et les modalités thérapeutiques. 


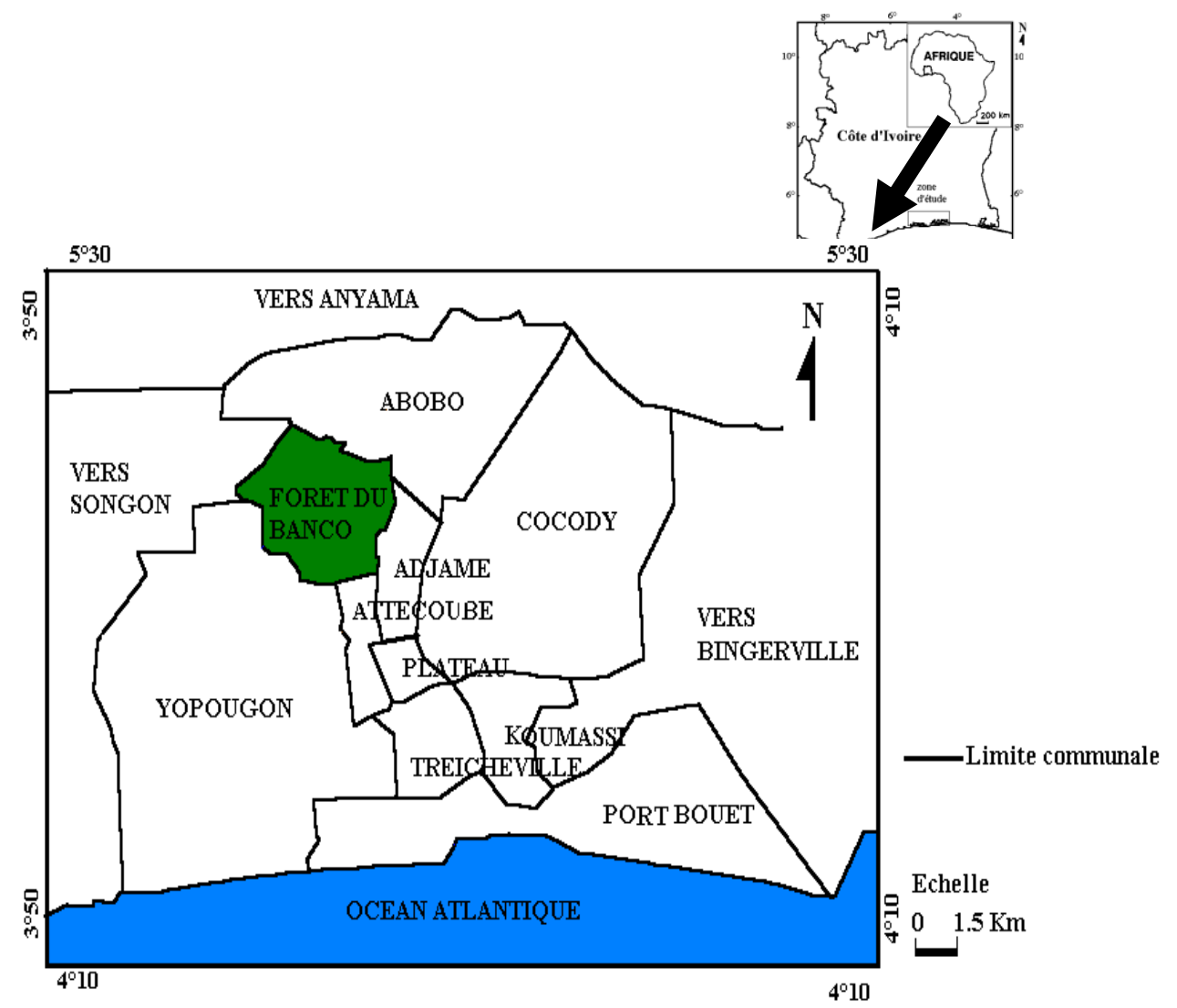

Figure 1: Carte de localisation la zone d'étude dans le district d'Abidjan.

Tableau 1: Répartition des enquêtés dans la zone d'étude.

\begin{tabular}{cccc}
\hline \multirow{2}{*}{ Communes } & \multicolumn{2}{c}{ Nombre d'enquêtés } & \multirow{2}{*}{ Sous-total } \\
\cline { 2 - 3 } & Tradithérapeutes & Herboristes & \\
\hline Abobo & 8 & 9 & 17 \\
\hline Adjamé & 12 & 14 & 26 \\
\hline Attécoubé & 2 & 4 & 6 \\
\hline Cocody & 2 & 0 & 2 \\
\hline Koumassi & 6 & 4 & 10 \\
\hline Marcory & 2 & 0 & 2 \\
\hline Plateau & 2 & 0 & 2 \\
\hline Port-Bouët & 4 & 4 & 8 \\
\hline Treichville & 2 & 0 & 2 \\
\hline Yopougon & 6 & 2 & 8 \\
\hline Total & $\mathbf{4 6}$ & $\mathbf{3 7}$ & $\mathbf{8 3}$ \\
\hline
\end{tabular}




\section{RESULTATS}

\section{Profil socio-démographique des} tradithérapeutes et herboristes enquêtés

L'étude a été menée auprès de 83 acteurs de la médecine traditionnelle, 46 tradithérapeutes et 37 herboristes dont les caractéristiques socio-démographiques sont consignées dans le Tableau 2.

Ces acteurs interrogés sont aussi bien de sexe masculin que féminin. Les tradithérapeutes interrogés sont majoritairement des hommes $(78,26 \%)$, tandis que les herboristes interrogés sont à majorité des femmes $(89,18 \%)$. La tranche d'âge la plus représentée pour les deux catégories d'acteurs est la tranche d'âge] 45-60] avec $59,03 \%$ des enquêtés. Les différentes personnes enquêtées sont majoritairement sans niveau d'instruction $(54,22 \%)$. Des proportions non négligeables de niveau primaire $(28,91 \%)$ et secondaire $(12,04 \%)$ ont été enregistrées lors des enquêtes. Elles appartiennent à quatre groupes ethniques (Akan, Mandé, Gour, Krou) parmi lesquels les mandés $(51,80 \%)$ et les Akans $(42,16 \%)$ sont les plus représentés.

\section{Diversité des plantes utilisées dans le traitement du paludisme}

Cette étude a permis de répertorier 54 espèces de plantes qui se répartissent entre 47 genres et 29 familles botaniques. Les plantes recensées et leurs indications thérapeutiques sont consignées dans le Tableau 3. Les familles les mieux représentées sont les Rubiaceae (5 espèces), Combretaceae (5 espèces), les Fabaceae (4 espèces), les Meliaceae (4 espèces), les Asteraceae (3 espèces), les Phyllanthaceae ( 3 espèces) et les Apocynaceae (3 espèces).

\section{Types morphologiques}

Les plantes inventoriées sont composées de 32 espèces d'arbustes et arbustes sarmenteux, de 12 espèces d'arbres, 4 espèces lianescentes et 6 herbacées. Quant aux types biologiques, les microphanérophytes (mp) avec 35 espèces $(64,81 \%)$, les nanophanérophytes (np) avec 11 espèces $(20,37 \%)$ et les hémicriptophytes avec 6 espèces $(11,11 \%)$ sont les plus représentés.
Les phanérophytes sont donc les plus abondantes avec $85,18 \%$.

\section{Types biogéographiques}

Les espèces des régions phytogéographiques Soudano-Zambézienne et Guinéo-Congolaise (GC-SZ) sont les plus représentées avec 26 espèces soit $48,15 \%$. Elles sont suivies des espèces de la région phytogéographique Guinéo-Congolaise (GC) et des espèces de la région phytogéographique Soudano-Zambézienne (SZ) avec chacune 11 espèces soit $20,37 \%$. Les espèces introduites (i), avec 5 espèces soit $9,26 \%$, sont les moins représentées.

\section{Fréquences de citation}

Les espèces les plus citées par les tradithérapeutes et les herboristes pour le traitement du paludisme sont, par ordre décroissant des fréquences de citation, Morinda lucida ( $\mathrm{FC}=7,65 \%)$, Cochlospermum planchonii $\quad(\mathrm{FC}=4,47 \%), \quad$ Combretum micranthum $\quad(\mathrm{FC}=4,47 \%), \quad$ Alchornea cordifolia $\quad(\mathrm{FC}=3,89 \%), \quad$ Harungana madagascariensis $\quad(\mathrm{FC}=3,89 \%)$, Hymenocardia acida $(\mathrm{FC}=3,61 \%)$ et Olax subscorpioidea $(\mathrm{FC}=3,46 \%)$ (Tableau 4).

\section{Contribution dans les recettes}

Les plantes recensées interviennent dans 104 recettes médicamenteuses dont $22,12 \%$ sont composées de recettes monospécifiques et $77,88 \%$ de recettes plurispécifiques. Les espèces les plus utilisées par les tradithérapeutes et les herboristes dans les différentes recettes pour le traitement du paludisme, par ordre décroissant des fréquences de contribution, sont: Alchornea cordifolia ( $\mathrm{CPr}=11,54 \%)$, Alstonia boonei ( $\mathrm{CPr}=9,60 \%), \quad$ Piliostigma thonningii $(\mathrm{CPr}=9,60 \%)$, Cissus doeringii $(\mathrm{CPr}=9,60 \%)$, Momordica charantia ( $\mathrm{CPr}=9,60 \%)$, Olax subscorpioidea $(\mathrm{CPr}=9,60 \%), \quad$ Harungana madagascariensis $(\mathrm{CPr}=8,65 \%)$, Combretum micranthum ( $\mathrm{CPr}=7,69 \%)$ et Sarcocephalus latifolius $(\mathrm{CPr}=7,69 \%) \quad($ Tableau 4). Le Tableau 5 présente quelques recettes plurispécifiques des espèces ayant les CPr les plus élevées.

\section{Indice de Confirmation}

L'indice de consensus n'est proche de 1 pour aucune des espèces de plantes 
recensées. Les espèces ayant les indices de consensus les plus élevés sont par ordre décroissant d'indice : Sarcocephalus latifolius Morinda lucida ( $\mathrm{ICs}=0,35$ ), Cochlospermum planchonii $\quad(\mathrm{ICs}=0,25), \quad$ Sarcocephalus latifolius $\quad(\mathrm{ICs}=0,22), \quad$ Harungana madagascariensis (ICs=0,19), Cochlospermum tinctorium (ICs $=0,19)$, Hymenocardia acida (ICs=0,19). Toutes les autres espèces ont des ICs faibles, en dessous de 0,19 (Tableau 4).

\section{Organes des plantes utilisés}

Les organes végétaux (drogues) utilisés par les tradithérapeutes et les herboristes sont les feuilles $(68,89 \%)$, les racines $(13,96 \%)$, les écorces de tiges $(11,92 \%)$, et la plante entière $(3,79 \%)$, les graines $(1,16 \%)$ ou la sève
$(0,29 \%)$. Les feuilles sont de loin les organes les plus utilisés dans les recettes suivies de la racine puis de l'écorce de tige (Figure 2).

\section{Modes de préparation des recettes}

Les modes de préparation utilisés par les tradithérapeutes et les herboristes sont : la décoction $(76,97 \%)$, la macération $(18,09 \%)$ et l'infusion $(4,94 \%)$ (Figure 3).

Voies d'administration des préparations médicamenteuses

La plupart des préparations sont administrées par voie orale $(84,04 \%)$ sous forme de boisson avec quelques fois l'usage de la voie rectale $(7,98 \%)$ par purge et de la voie externe $(7,98 \%)$ par bain (Figure 4$)$.

Tableau 2: Caractéristiques socio-démographiques des tradithérapeutes et herboristes enquêtés.

\begin{tabular}{llccc}
\hline & & \multicolumn{2}{c}{ Personnes enquêtées } & Moyennes (\%) \\
\cline { 3 - 4 } Genre & Paramètres & Tradithérapeutes (\%) & Herboristes (\%) & 48,19 \\
& Homme & $\mathbf{7 8 , 2 6}$ & 10,8 & 51,81 \\
\hline Niveau & Femme & 21,7 & $\mathbf{8 9 , 1 8}$ & $54,, 22$ \\
d'étude & Analphabète & 45,7 & 64,9 & 28,92 \\
& Primaire & 26,0 & 32,4 & 12,05 \\
& Secondaire & 19,5 & 2,7 & 4,81 \\
\hline Classe d'âge & Supérieur & 8,7 & 0 & 3,62 \\
& $<30$ ans & 0 & 8,1 & 26,51 \\
& {$[30-45]$} & 19,5 & 32,4 & $\mathbf{5 9 , 0 3}$ \\
& ] $45-60]$ & 60,9 & 56,7 & 10,84 \\
& $>60$ ans & 19,5 & 0 & $\mathbf{4 2 , 1 6}$ \\
\hline \multirow{2}{*}{ ethniques } & Akan & 52,2 & 29,7 & 2,41 \\
& Gour & 2,2 & 2,7 & 1,20 \\
& Krou & 2,2 & 0 & $\mathbf{5 1 , 8 0}$ \\
& Mandé & 39,1 & 64,9 & 2,41 \\
\hline
\end{tabular}

Les valeurs en gras représentent les valeurs des paramètres sociodémographiques les plus caractéristiques. 
Tableau 3: Fréquences de citation, Contribution des espèces et Indice de Consensus des plantes antipaludiques recensées dans le district d'Abidjan.

\begin{tabular}{|c|c|c|c|c|c|c|c|}
\hline Espèces végétales & $\begin{array}{l}\text { FC } \\
(\%)\end{array}$ & $\begin{array}{l}\mathbf{C P r} \\
(\%)\end{array}$ & ICs & Espèces végétales & $\begin{array}{l}\text { FC } \\
(\%)\end{array}$ & $\begin{array}{l}\mathrm{CPr} \\
(\%)\end{array}$ & ICs \\
\hline Morinda lucida & 7,65 & 17,3 & $\mathbf{0 , 3 5}$ & Terminalia laxiflora & 1,59 & 5,77 & 0,08 \\
\hline Cochlospermum planchonii & 4,47 & 8,65 & $\mathbf{0 , 2 5}$ & Guiera seneglensis & 1,44 & 3,85 & 0,07 \\
\hline Combretum micranthum & 4,18 & 7,69 & 0,17 & Catharanthus roseus & 1,29 & 3,85 & 0,07 \\
\hline Alchornea cordifolia & 3,89 & 11,54 & 0,2 & Annona muricata & 1,15 & 0,96 & 0,09 \\
\hline $\begin{array}{l}\text { Harungana } \\
\text { madagascariensis }\end{array}$ & 3,89 & 8,65 & 0,19 & Mitragyna inermis & 1,15 & 1,92 & 0,08 \\
\hline Hymenocardia acida & 3,61 & 9,61 & 0,19 & Paullina pinnata & 1,15 & 3,84 & 0,07 \\
\hline Olax subscorpioidea & 3,46 & 9,61 & 0,12 & Vernonia amygdalina & 1,15 & 0,96 & 0,09 \\
\hline Alstonia boonei & 3,03 & 9,61 & 0,13 & Azadirachta indica & 1,01 & 1,92 & 0,08 \\
\hline Cissus doeringii & 2,74 & 9,61 & 0,16 & Crossopteryx febrifuga & 1,01 & 1,92 & 0,05 \\
\hline Hoslundia opposita & 2,74 & 7,69 & 0,13 & Khaya senegalensis & 1,01 & 0,96 & 0,07 \\
\hline Momordica charantia & 2,74 & 9,61 & 0,1 & Pavetta crassipes & 1,01 & 2,88 & 0,05 \\
\hline Sarcocephalus latifolius & 2,74 & 7,69 & 0,22 & Rauvolfia vomitoria & 1,01 & 1,92 & 0,07 \\
\hline Anthocleista djalonensis & 2,45 & 5,77 & 0,12 & Lippia multiflora & 0,86 & 1,92 & 0,05 \\
\hline Anthocleista nobilis & 2,45 & 1,92 & 0,11 & Aspilia africana & 0,72 & 1,92 & 0,05 \\
\hline Carapa procera & 2,45 & 4,81 & 0,05 & Bridelia ferruginea & 0,72 & 0,96 & 0,06 \\
\hline Senna sieberiana & 2,45 & 8,65 & 0,13 & Ximenia americana & 0,72 & 0,96 & 0,05 \\
\hline Phyllanthus amarus & 2,31 & 4,81 & 0,12 & Senna alata & 0,58 & 0,96 & 0,05 \\
\hline Piliostigma thonningii & 2,31 & 9,61 & 0,13 & Combretum molle & 0,58 & 1,92 & 0,05 \\
\hline Vernonia colorata & 2,31 & 3,85 & 0,12 & Cymbogopon citratus & 0,58 & 2,88 & 0,05 \\
\hline Senna occidentalis & 2,16 & 3,84 & 0,09 & Diospyros mespiliformis & 0,58 & 1,92 & 0,05 \\
\hline Cochlospermum tinctorium & 2,16 & 0,96 & 0,19 & Eucalyptus camaldulensis & 0,58 & 2,88 & 0,08 \\
\hline Trema orientalis & 2,02 & 1,92 & 0,13 & Jatropha curcas & 0,58 & 0,96 & 0,05 \\
\hline Annona senegalensis & 1,87 & 2,88 & 0,09 & Lantana camara & 0,58 & 0,96 & 0,05 \\
\hline Anogeissus leiocarpus & 1,87 & 2,88 & 0,08 & $\begin{array}{l}\text { Securidaca } \\
\text { longepedunculata }\end{array}$ & 0,58 & 0,96 & 0,05 \\
\hline Trichilia emetica & 1,73 & 3,84 & 0,09 & Solanum nigrum & 0,58 & 0,96 & 0,05 \\
\hline Carica papaya & 1,59 & 3,85 & 0,07 & Cnestis ferruginea & 0,43 & 1,92 & 0,02 \\
\hline Mangifera indica & 1,59 & 2,88 & 0,08 & Vitellaria paradoxa & 0,43 & 1,92 & 0,02 \\
\hline
\end{tabular}

$\mathrm{FC}=$ fréquence de citation, $\mathrm{Cpr}=$ Contribution de chaque espèce aux recettes médicamenteuses, $\mathrm{ICs}=$ indice de confirmation

Les valeurs en gras représentent les valeurs élevées pour chaque paramètre ethnobotanique 
Tableau 4 : Plantes utilisées contre le paludisme dans le district d'Abidjan.

\begin{tabular}{|c|c|c|c|c|c|c|c|}
\hline $\mathbf{N}^{\circ}$ & Espèces végétales & Familles & $\begin{array}{l}\text { Type biologique } \\
\text { et chorologie }\end{array}$ & $\begin{array}{l}\text { Organe } \\
\text { utilisé }\end{array}$ & $\begin{array}{l}\text { Mode de } \\
\text { préparation }\end{array}$ & $\begin{array}{l}\text { Mode } \\
\text { d'administration }\end{array}$ & $\begin{array}{l}\text { Posologie } \\
\text { Des recettes }\end{array}$ \\
\hline 1 & Alchornea cordifolia (Schum et Thonn.) ${ }^{1}$ & Euphorbiaceae & GC-SZ, mp, bl & $\mathrm{F}, \mathrm{R}$ & Déc & oral, bain & 1 verre 3 fois par jour \\
\hline 2 & Alstonia boonei De Wild. ${ }^{1}$ & Apocynaceae & $\mathrm{GC}, \mathrm{Mp}, \mathrm{a}$ & $\mathrm{F}, \mathrm{E}$ & Déc, Mac & oral & 1 verre 3 fois par jour \\
\hline 3 & Annona muricata L. & Annonaceae & i, np (Hpy), b & $\mathrm{F}$ & Déc & oral, bain & $\begin{array}{l}\text { En association, } 1 \text { verre } 3 \text { fois par } \\
\text { jour+Bain }\end{array}$ \\
\hline 4 & Annona senegalensis Pers & Annonaceae & SZ, np (Hpy), b & $\mathrm{F}$ & Déc & oral, bain & $\begin{array}{l}\text { En association, } 1 \text { verre } 3 \text { fois par } \\
\text { jour+Bain }\end{array}$ \\
\hline 5 & Anogeissus leiocarpus (DC.) Guill. \& Perr. & Combretaceae & $\mathrm{SZ}, \mathrm{mp}, \mathrm{a}$ & $\mathrm{F}, \mathrm{R}$ & Déc & oral, bain & $\begin{array}{l}\text { En association, } 1 \text { verre } 3 \text { fois par } \\
\text { jour+Bain }\end{array}$ \\
\hline 6 & Anthocleista djalonensis A. Chew ${ }^{1}$ & Gentianaceae & GC-SZ, mp, a & $\mathrm{F}, \mathrm{R}, \mathrm{E}$ & Déc, Mac & oral & $1 / 2$ verre 3 fois par jour \\
\hline 7 & Anthocleista nobilis G. Don ${ }^{1}$ & Gentianaceae & GCW, mp, a & $\mathrm{F}, \mathrm{R}, \mathrm{E}$ & Déc, Mac & oral & $1 / 2$ verre 3 fois par jour \\
\hline 8 & Aspilia africana (Pers) CD. Adams & Asteraceae & $\mathrm{GC}, \mathrm{np}, \mathrm{h}$ & $\mathrm{F}$ & Déc & oral & En association, 1 verre 3 fois par jour \\
\hline 9 & Azadirachta indica A.Juss 1 & Meliaceae & $\mathrm{i}, \mathrm{mp}, \mathrm{a}$ & $\mathrm{F}, \mathrm{E}$ & Déc, Mac & oral & $1 / 2$ verre 3 fois par jour \\
\hline 10 & Bridelia ferruginea Benth $^{1}$ & Phyllanthaceae & GC-SZ, mp, b & $\mathrm{F}, \mathrm{E}, \mathrm{R}$ & Déc, Mac & oral & $1 / 2$ verre 3 fois par jour \\
\hline 11 & Carapa procera DC. & Meliaceae & GC-SZ, mp, a & $\mathrm{F}, \mathrm{E}$ & Déc & oral & En association, 1 verre 3 fois par jour \\
\hline 12 & Carica papaya $\mathrm{L}^{1}$. & Caricaceae & $\mathrm{GC}, \mathrm{mp}, \mathrm{h}$ & $\mathrm{F}$ & Déc, Mac & oral & 1 verre 3 fois par jour + Bain \\
\hline 13 & Senna alata $\mathrm{L}$. & Fabacaceae & $\mathrm{GC}, \mathrm{np}, \mathrm{b}$ & $\mathrm{F}$ & Déc & oral & En association, 1 verre 3 fois par jour \\
\hline 14 & Senna occidentalis L. & Fabacaceae & GC-SZ, np, bl & $\mathrm{F}, \mathrm{G}$ & Déc, Inf & oral & $\begin{array}{l}\text { En association, } 1 \text { verre } 3 \text { fois par } \\
\text { jour+Bain }\end{array}$ \\
\hline 15 & Senna sieberiana DC. ${ }^{1}$ & Fabacaceae & GC-SZ, mp, b & $\mathrm{F}, \mathrm{R}$ & Déc, Mac & oral, rectal & $1 / 2$ verre 3 fois par jour +purge \\
\hline 16 & Catharanthus roseus (L.) G. Don $^{1}$ & Apocynaceae & $\mathrm{GC}, \mathrm{np}, \mathrm{h}$ & $\mathrm{F}, \mathrm{R}$ & Déc, Mac & oral & $1 / 2$ verre 3 fois par jour \\
\hline 17 & Cissus doeringii Gilg \& M.Brandt & Vitaceae & GC-SZ,np(Hpy),1 & $\mathrm{F}, \mathrm{R}$ & Déc & oral & $\begin{array}{l}\text { En association, } 1 / 2 \text { verre } 3 \text { fois par } \\
\text { jour }\end{array}$ \\
\hline 18 & Cnestis ferruginea DC. & Connaraceae & GC, Lmp, bl & $\mathrm{F}$ & Déc & oral & $\begin{array}{l}\text { En association, } 1 \text { verre } 3 \text { fois par } \\
\text { jour+Bain }\end{array}$ \\
\hline
\end{tabular}


Y. SYLLA et al. / Int. J. Biol. Chem. Sci. 12(3): 1380-1400, 2018

\begin{tabular}{|c|c|c|c|c|c|c|}
\hline 19 Cochlospermum planchonii Hook.f. & Bixaceae & SZ, np (Hpy), bl & $\mathrm{F}$ & Déc & oral & 1 verre 3 fois par jour \\
\hline 20 Cochlospermum tinctorium Perr.ex.A. Rich. ${ }^{1}$ & Bixaceae & SZ, np (Hpy), bl & $\mathrm{F}$ & Déc & oral & 1 verre 3 fois par jour \\
\hline $21 \quad$ Combretum micranthum G. Don ${ }^{1}$ & Combretaceae & $\mathrm{SZ}, \mathrm{mp}, \mathrm{b}$ & $\mathrm{F}$ & Déc & oral & 1 verre 3 fois par jour \\
\hline 22 Combretum molle R.Br.ex G. Don ${ }^{1}$ & Combretaceae & $\mathrm{SZ}, \mathrm{mp}, \mathrm{b}$ & $\mathrm{F}$ & Déc & oral & 1 verre 3 fois par jour \\
\hline $\begin{array}{l}23 \text { Crossopteryx febrifuga. } \\
\text { (Afsel.ex.G.Don)Benth }\end{array}$ & Rubiaceae & GC-SZ, mp, b & $\mathrm{F}, \mathrm{R}$ & Déc & oral, rectal & $\begin{array}{l}\text { En association, } 1 / 2 \text { verre } 3 \text { fois par } \\
\text { jour+Purge }\end{array}$ \\
\hline 24 Cymbogopon citratus (DC.) Stapf & Poaceae & GC-SZ, H, h & $\mathrm{F}, \mathrm{P}$ & Déc, Inf & oral & En association, 1 verre 3 fois par jour \\
\hline 25 Diospyros mespiliformis Hochst.ex DC. & Ebenaceae & GC-SZ, mp, a & $\mathrm{F}$ & Déc & oral & En association, 1 verre 3 fois par jour \\
\hline 26 Eucalyptus camaldulensis Dehnh & Myrtaceae & $\mathrm{i}, \mathrm{mp}, \mathrm{a}$ & $\mathrm{F}$ & Déc & oral & $\begin{array}{l}\text { En association, } 1 \text { verre } 3 \text { fois par } \\
\text { jour+Bain }\end{array}$ \\
\hline 27 Guiera seneglensis J.F. Gmel & Combretaceae & $\mathrm{SZ}, \mathrm{np}, \mathrm{b}$ & $\mathrm{F}, \mathrm{E}, \mathrm{R}$ & Déc, Mac & oral & $1 / 2$ verre 3 fois par jour + Bain +purge \\
\hline 28 Harungana madagascariensis Auct. & Hypericaceae & $\mathrm{GC}, \mathrm{mp}, \mathrm{b}$ & $\mathrm{F}$ & Déc & oral & 1 verre 3 fois par jour + Bain +purge \\
\hline 29 Hoslundia opposita Vahl. & Lamiaceae & GC-SZ, np, b & $\mathrm{F}$ & Déc & oral & 1 verre 3 fois par jour + Bain \\
\hline 30 Hymenocardia acida Tul. & Phyllanthaceae & GC-SZ, mp, b & $\mathrm{F}$ & Déc, Inf & oral & En association, 1 verre 3 fois par jour \\
\hline 31 Jatropha curcas L. & Euphorbiaceae & GC-SZ, np, b & $\mathrm{F}, \mathrm{S}$ & Déc & oral & En association, 1 verre 3 fois par jour \\
\hline 32 Khaya senegalensis (Desr.) A.Juss & Meliaceae & $\mathrm{GC}, \mathrm{mp}, \mathrm{b}$ & F, E, R & Déc & oral, rectal & $\begin{array}{l}\text { En association, } 1 \text { verre } 3 \text { fois par } \\
\text { jour+Purge }\end{array}$ \\
\hline Lantana camara L. & Verbenaceae & i, np, b & $\mathrm{F}$ & Déc, Inf & oral & En association, 1 verre 3 fois par jour \\
\hline Lippia multiflora Moldente & Verbenaceae & GC-SZ, np, b & $\mathrm{F}$ & Déc, Inf & oral & \\
\hline 35 Mangifera indica L. $^{1}$ & Anacardiaceae & $\mathrm{i}, \mathrm{mp}, \mathrm{a}$ & $F, E, R$ & Déc & oral, bain & $1 / 2$ verre 3 fois par jour + Bain \\
\hline 36 Mitragyna inermis (Willd.) K.Schum & Rubiaceae & $\mathrm{SZ}, \mathrm{mp}, \mathrm{b}$ & $\mathrm{F}$ & Déc & oral & En association, 1 verre 3 fois par jour \\
\hline Momordica charantia L. & Cucurbitaceae & GC, Th, 1 & $\mathrm{~F}, \mathrm{P}$ & Déc, Mac & oral & En association, 1 verre 3 fois par jour \\
\hline 38 Morinda lucida Benth. & Rubiaceae & GC-SZ, mp, b & $\mathrm{F}, \mathrm{E}$ & Déc & oral & $\begin{array}{l}\text { En association, } 1 \text { verre } 3 \text { fois par } \\
\text { jour+Bain }\end{array}$ \\
\hline 39 Sarcocephalus latifolius E.A.Bruce & Rubiaceae & GC-SZ, mp, bl & F, E, R & Déc, Mac & oral & $\begin{array}{l}\text { En association, } 1 / 2 \text { verre } 3 \text { fois par } \\
\text { jour+Bain }\end{array}$ \\
\hline 40 Olax subscorpioidea Oliv. & Olacaceae & GC-SZ, mp, b & $\mathrm{F}, \mathrm{R}$ & Décoction & oral & $\begin{array}{l}\text { En association, } 1 \text { verre } 3 \text { fois par } \\
\text { jour+Bain }\end{array}$ \\
\hline
\end{tabular}


Y. SYLLA et al. / Int. J. Biol. Chem. Sci. 12(3): 1380-1400, 2018

\begin{tabular}{|c|c|c|c|c|c|c|}
\hline 41 Paullina pinnata $\mathrm{L}$. & Sapindaceae & GC-SZ, mp, 1 & $\mathrm{~F}$ & Déc & oral & $\begin{array}{l}\text { En association, } 1 \text { verre } 3 \text { fois par } \\
\text { jour+Bain }\end{array}$ \\
\hline 42 Pavetta crassipes K. Schum. & Rubiaceae & GC-SZ, mp, h & $\mathrm{F}, \mathrm{P}$ & Déc, Inf & oral & $\begin{array}{l}\text { En association, } 1 \text { verre } 3 \text { fois par } \\
\text { jour+Bain }\end{array}$ \\
\hline 43 Phyllanthus amarus Schumach. \&Thonn. ${ }^{1}$ & Phyllanthaceae & GC,np, h & $\mathrm{F}, \mathrm{P}$ & Déc, Inf & oral & 1 verre 3 fois par jour \\
\hline 44 Piliostigma thonningii (Schum.) Milne-Red. & Fabaceae & GC-SZ, mp, b & $\mathrm{F}$ & Déc & oral & $\begin{array}{l}\text { En association, } 1 \text { verre } 3 \text { fois par } \\
\text { jour+Bain }\end{array}$ \\
\hline 45 Rauvolfia vomitoria Afze. & Apocynaceae & GC-SZ, mp, b & F S & Déc & oral & $1 / 2$ verre 3 fois par jour \\
\hline 46 Securidaca longepedunculata L. (Fresen.) & Polygalaceae & $\mathrm{SZ}, \mathrm{mp}, \mathrm{b}$ & $\mathrm{F}$ & Déc & oral & En association, 1 verre 3 fois par jour \\
\hline 47 Solanum nigrum $\mathrm{L}^{.1}$ & Solanaceae & GC-SZ, np, h & $\mathrm{F}, \mathrm{P}$ & Déc, Inf & oral & 1 verre 3 fois par jour \\
\hline 48 Terminalia laxiflora Engl. \& Diesels & Combretaceae & $\mathrm{SZ}, \mathrm{np}, \mathrm{mp}$ & $\mathrm{F}$ & Déc & oral, bain & $\begin{array}{l}\text { En association, } 1 \text { verre } 3 \text { fois par } \\
\text { jour+Bain }\end{array}$ \\
\hline 49 Trema orientalis (L.) Blume & Cannabaceae & GC-SZ, b & $\mathrm{F}$ & Déc & oral & En association, 1 verre 3 fois par jour \\
\hline 50 Trichilia emetica Vahl & Meliaceae & $\mathrm{SZ}, \mathrm{mp}, \mathrm{b}$ & $\mathrm{F}$ & Déc & oral & En association, 1 verre 3 fois par jour \\
\hline 51 Vernonia amygdalina Delile $^{1}$ & Asteraceae & GC-SZ, mp, b & $\mathrm{F}$ & Déc, Mac & oral & 1 verre 3 fois par jour \\
\hline 52 Vernonia colorata (Willd) Drake ${ }^{1}$ & Asteraceae & GC-SZ, mp, b & $\mathrm{F}$ & Déc, Mac & oral & 1 verre 3 fois par jour \\
\hline 53 Vitellaria paradoxa C.F. Gaertn & Sapotaceae & $\mathrm{GC}, \mathrm{mp}, \mathrm{a}$ & $\mathrm{F}$ & Déc & oral & En association, 1 verre 3 fois par jour \\
\hline 54 Ximenia americana L. & Ximeniaceae & GC-SZ, mp, b & F, E, R & Déc, Mac & oral, rectal & 1 verre 3 fois par jour+Purge \\
\hline
\end{tabular}


Tableau 5: Compositions et posologies des recettes plurispécifiques des espèces ayant des Contributions aux recettes les plus élevées (CPr $\geq 4)$.

\begin{tabular}{|c|c|c|c|c|c|c|c|c|c|c|c|c|c|c|c|c|c|c|c|c|c|c|c|c|c|c|c|c|c|}
\hline Espèces végétaltes & 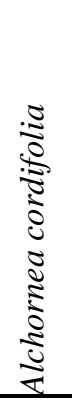 & 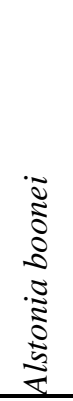 & 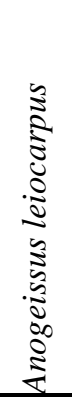 & 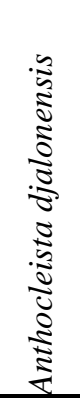 & 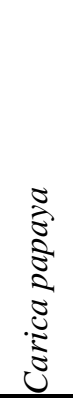 & 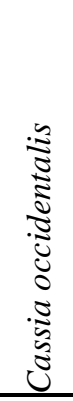 & 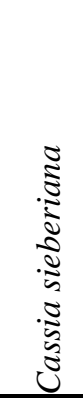 & 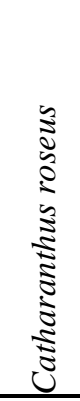 & 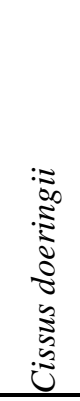 & 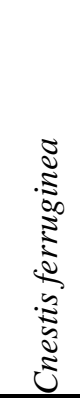 & 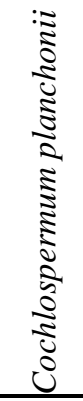 & 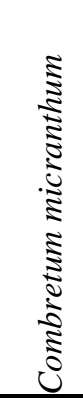 & 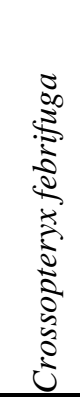 & 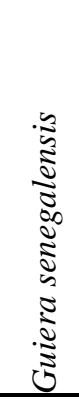 & 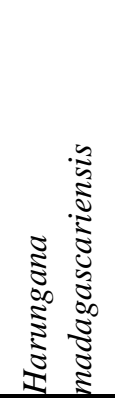 & 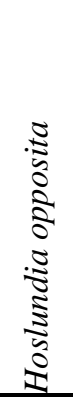 & 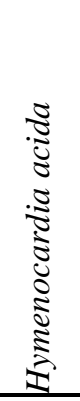 & 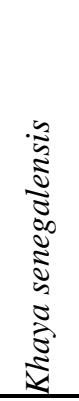 & 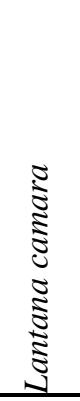 & 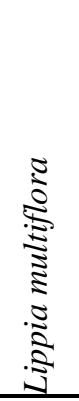 & 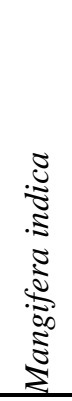 & 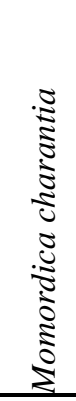 & 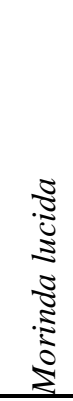 & 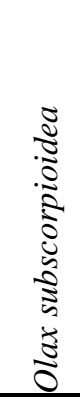 & 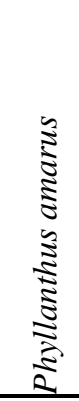 & 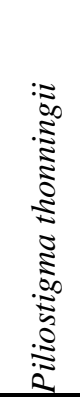 & 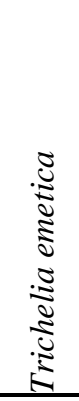 & 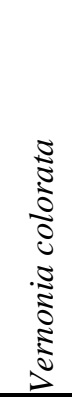 & Posologies \\
\hline Alchornea cordifolia & & $\mathrm{xx}$ & & $\mathrm{xx}$ & & & & & & & & & & & & & & & & & & & & & & & & $\mathrm{xx}$ & $\begin{array}{l}\text { décocté des } \\
\text { feuilles ; boire } 1 \\
\text { verre } 3 \text { fois par } \\
\text { jour + Bain } \\
+ \text { purge }\end{array}$ \\
\hline Alstonia boonei & & & & & $\mathrm{xx}$ & & & & & & & & & & & & & & & & & & & & & & & & $\begin{array}{l}\text { décocté des } \\
\text { feuilles et/ou } \\
\text { d'écorces de tige, } \\
\text { boire } 1 / 2 \text { verre } 3 \\
\text { fois par jour }\end{array}$ \\
\hline Anogeissus leiocarpus & & & & & & & & & & & $\mathrm{xx}$ & & & & & & & & & & & & & & & $\mathrm{xx}$ & & & $\begin{array}{l}\text { décocté des } \\
\text { feuilles, boire } 1 \\
\text { verre } 3 \text { fois par } \\
\text { jour }\end{array}$ \\
\hline $\begin{array}{l}\text { Anthocleista } \\
\text { djalonensis }\end{array}$ & $\mathrm{xx}$ & & & & $\mathrm{xx}$ & & & & & & & & & & & & & & & & & & & & & & & & $\begin{array}{l}\text { décocté des } \\
\text { feuilles, boire } 1 / 2 \\
\text { verre } 3 \text { fois par }\end{array}$ \\
\hline
\end{tabular}


jour + Bain décocté des

feuilles ou

d'écorces de tige;

boire $1 / 2$ verre 3

fois par jour

+Bain

macération dans

l'eau après

trituration des

feuilles ou

infusion des

feuilles; boire 1

verre 3 fois par

décocté des

racines de $S$.

sieberiana +

feuilles de

C.planchonii et

de $P$. .thonningii

; boire $1 / 2$ verre

3 fois par jour

décocté des

feuilles, boire 1

verre 3 fois par 
racines de $S$, sieberiana et de A, leiocarpus + feuilles $d e P$, thonningii; boire $1 / 2$ verre 3 fois

par

jour+Bain+Purge décocté des

feuilles ; boire 1 verre 3 fois par jour

décocté des feuilles ; boire 1 verre 3 fois par jour + Bain infusé ou décocté des feuilles ; boire 1 verre 3 fois par jour décocté des

feuilles, boire 1 verre 3 fois par jour décocté des

feuilles, boire verre 3 fois par jour + Bain 
décocté des

feuilles, boire 1

verre 3 fois par

jour

décocté des

feuilles + racine

de A, boonei,

boire 1 verre 3

fois par jour

décocté des

feuilles, boire 1

verre 3 fois par

Momordica charantia $\mathrm{xx}$

$\mathrm{xx}$

jour

décocté des

feuilles ou/et de

racines, boire $1 / 2$

verre 3 fois par

Morinda lucida

$\mathrm{xx}$

$\mathrm{XX}$

$\mathrm{Xx}$

jour

décocté des

feuilles, boire $1 / 2$

verre 3 fois par

Olax subscorpioidea

XX

$\mathrm{xx}$ jour

infusé ou décocté

des feuilles, boire

1 verre 3 fois par

jour 
1 verre 3 fois par jour

décocté des

feuilles, boire 1

Sarcocephalus verre 3 fois par

latifolius $\mathrm{xx}$ jour

décocté des

feuilles et/ou de racines, boire $1 / 2$ verre 3 fois par jour +Bain décocté des

feuilles, boire 1 verre 3 fois par jour + Bain décocté des

feuilles et ou de racines, boire $1 / 2$ verre 3 fois par jour

macération dans

l'eau après

trituration des

feuilles; boire 1

verre 3 fois par

jour 


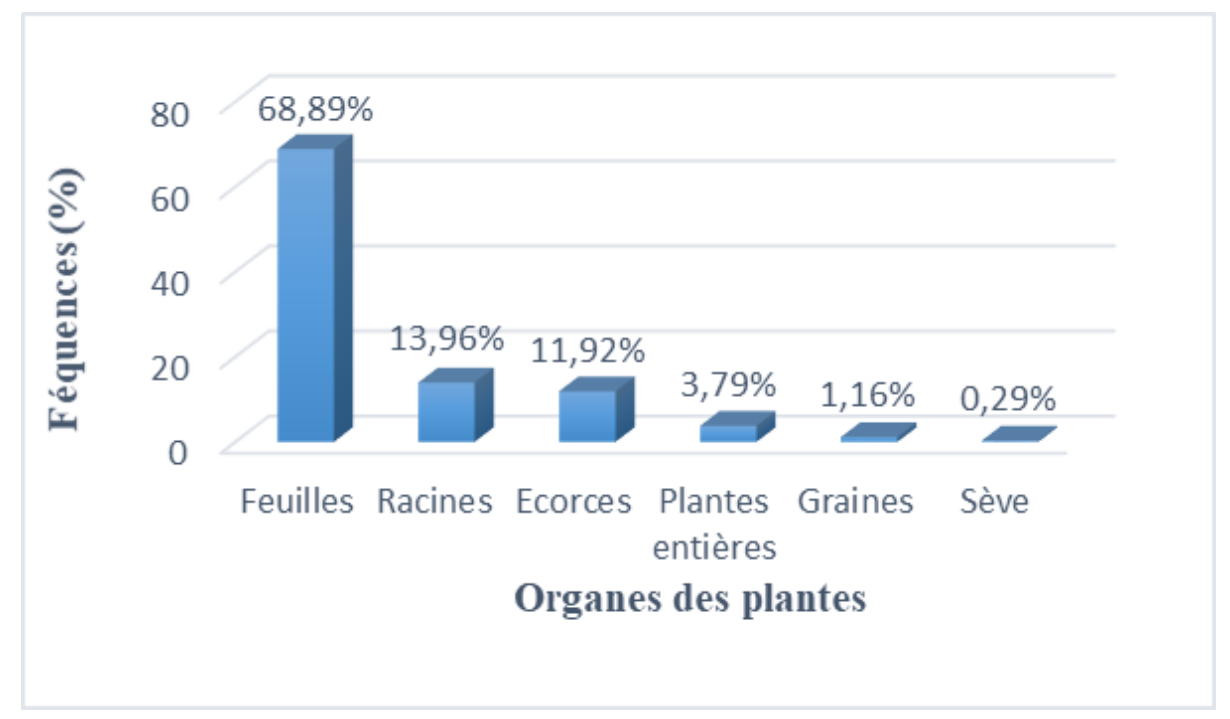

Figure 2: Fréquences de citation d'utilisation des organes de plantes.

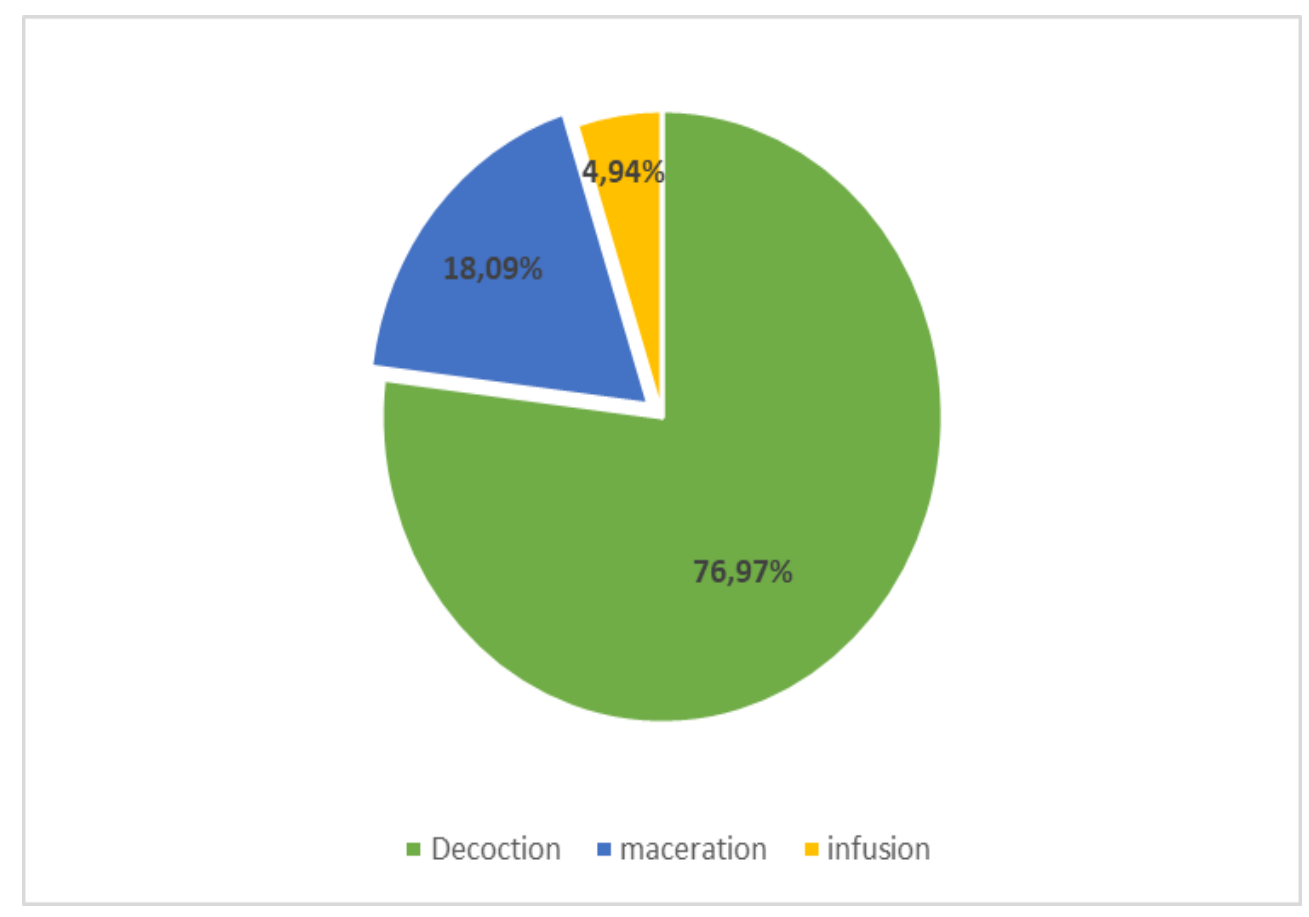

Figure 3: Fréquence de citation des modes de préparation des drogues. 


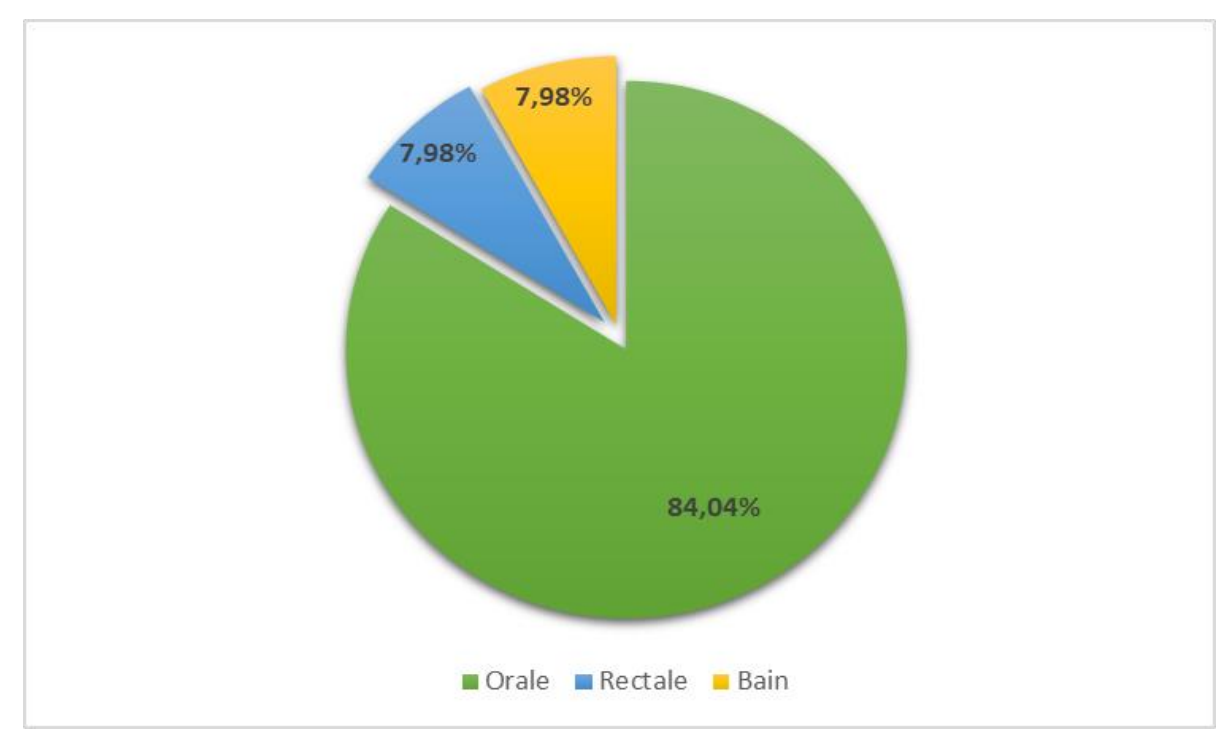

Figure 4: Fréquence de citation des modes d'administration des préparations médicamenteuses

\section{DISCUSSION}

La présente étude a consisté à répertorier les plantes utilisées par les tradithérapeutes et herboristes pour traiter le paludisme dans 10 communes du district autonome d'Abidjan. Elle a été menée auprès de 83 acteurs de la médecine traditionnelle dont 46 tradithérapeutes et 37 herboristes. Elle a montré que les tradithérapeutes interrogés sont majoritairement des hommes tandis que les herboristes sont à majorité des femmes. La prédominance des femmes parmi les herboristes a été déjà rapportée dans des études similaires réalisées en Côte d'Ivoire par Ambé (2016) sur des marchés d'Abidjan avec $96,00 \%$ de femmes et par Hermans et al. (2004) sur des marchés au sud du Bénin avec pratiquement $100 \%$ de femmes. Cela s'explique par le fait qu'en Côte d'Ivoire, la vente dans les marchés est une activité habituellement investie par les femmes. Par contre, sur les marchés de Dakar, au Sénégal (Dasylva, 2001), au Burkina-Faso (Sanon et al., 2002) et au Mali (Togola et al., 2005), les hommes sont les plus nombreux dans le commerce des plantes. Cela pourrait s'expliquer par la différence de l'environnement et des traits socioculturels entre les populations de ces contrées et les populations du district d'Abidjan.

Les deux catégories d'acteurs interrogées sont pour la plupart âgés de plus de 40 ans parce que la connaissance des usages des plantes médicinales et leurs propriétés sont généralement acquises suite à de longues années d'expériences et transmises d'une génération à l'autre (Benlamdini et al., 2014).

Parmi les espèces répertoriées, les phanérophytes sont les plus représentés $(85,81 \%)$. Ces résultats reflètent l'état de la végétation des zones tropicales et équatoriales, dont la proportion en phanérophytes est estimée entre 80 et $90 \%$ (Ambé, 2006). Les plantes ligneuses sont les plus sollicitées (81,48\%). Selon Adomou et al. (2012), cette sollicitation se justifierait par le caractère pérenne de ces espèces permettant de disposer d'au moins un organe en chaque saison. Concernant la chorologie, les espèces de la région Guinéo-congolaise et SoudanoZambézienne sont les plus importantes $(48,15 \%)$, se superposant ainsi aux résultats obtenus par Ambé (2016) dans les marchés d'Abidjan.

Dans cette étude, les familles les plus représentées sont les Rubiaceae $(9,25 \%)$, les 
Combretaceae $(7,41 \%)$, les Fabaceae $(7,41 \%)$, les Meliaceae $(7,41 \%)$, les Asteraceae $(5,56 \%)$, les Phyllanthaceae $(5,56 \%)$ et les Apocynaceae $(5,56 \%)$. Ces résultats sont semblables, à quelques différences près, à ceux de Kipré et al. (2017) sur les plantes antipaludiques dans le département d'Agboville, localité proche d'Abidjan. Kipré et al. (2017) ont rapporté que les familles les plus représentées sont les Fabaceae $(8,80 \%)$; les Euphorbiaceae, les Rubiaceae (7,00\%), les Apocynaceae et les Meliaceae $(5,30 \%)$. La forte représentativité de ces familles a été également relevée dans des enquêtes ethnobotaniques réalisées dans d'autres régions du pays sur les plantes antipaludiques. Bla et al. (2015) ont mentionné comme familles les plus représentées, les Euphorbiaceae $(11,53 \%)$, les Rubiaceae $(7,69 \%)$, et les Fabaceae (7,69\%) lors des enquêtes menées à Toumodi. Ouattara (2006) a rapporté à Divo parmi les familles prépondérantes les Apocynaceae (25\%), les Euphorbiaceae $(8,92 \%)$, les Rubiaceae $(8,33 \%)$. Ces résultats s'expliqueraient par le fait que ces familles font partie, du point de vue générique et spécifique, des plus importantes de la flore ivoirienne (Ambé, 2006).

L'analyse des Fréquences de citation des espèces et de leur contribution dans les différentes recettes montre que Morinda lucida, Cochlospermum planchonii, Sarcocephalus latifolius, Alchornea cordifolia, Cochlospermum tinctorium, Harungana madagascariensis, Hymenocardia acida, Combretum micranthum et Cissus doeringii sont les espèces les plus citées et les plus utilisées dans les différentes recettes pour traiter le paludisme par les tradithérapeutes et herboristes à Abidjan. Cependant, la plupart des autres espèces recensées semblent être plus ou moins connues eu égard à leur fréquence de citation et à leur contribution dans les différentes recettes non négligeables. Elles ont, aussi, été rapportées par d'autres auteurs comme Zirihi (2006), N'Guessan et al. (2008), Yapi (2013), Bla et al. (2015), Kipré et al. (2017) ainsi que Dénou et al. (2017). Cette convergence des résultats avec ceux de ces auteurs témoignerait de la fiabilité des données obtenues dans ce travail. En revanche, les espèces comme Vitellaria paradoxa et Cnestis ferruginea se sont avérées très peu citées $(\mathrm{FC}=0,02 \%)$. D'autres espèces comme Cissus doeringii et Terminalia laxiflora, à ma connaissance, à ce jour sont très peu rapportées par dans les études ethnobotaniques comme plantes antipaludiques. Cela peut s'expliquer par le fait que ces plantes ont été gardées longtemps en secret ou que leurs vertus viennent d'être révélées. De telles drogues peuvent s'avérer très prometteuses. Par ailleurs, les faibles indices de consensus obtenus pour chacune des espèces, indiquent qu'il y a un faible accord autour de leur usage dans le traitement du paludisme. Cela s'expliquerait par la forte diversité culturelle des enquêtés qui appartiennent à des groupes socio-culturels et à des sphères géographiques différentes venus monnayer leurs savoirs dans cette métropole cosmopolite d'Abidjan.

Les feuilles sont les organes les plus utilisés $(68,89 \%)$, suivies des racines et des écorces de tige. La prédominance des feuilles dans les recettes pour le traitement du paludisme a été rapportée par d'autres auteurs comme Zirihi (2006), Bla et al. (2015) et Kipré et al. (2017). Cette fréquence d'utilisation élevée des feuilles s'expliquerait par l'aisance et la rapidité de récolte mais aussi et surtout par le fait que ces organes sont le siège de la photosynthèse et parfois le lieu de stockage des métabolites secondaires responsables des propriétés pharmacologiques de la plante (Mangambu et al., 2014). Les racines, avec $14 \%$ des cas, sont la deuxième catégorie d'organes la plus utilisée. Mais leur prélèvement supprime la possibilité de ravitaillement de la plante en éléments nutritifs, ce qui affecte son aspect végétatif ainsi que sa physiologie (Yapi, 2013).

La décoction aqueuse suivie de la macération et de l'infusion sont les modes de préparation les plus utilisés de ces organes. $\mathrm{La}$ décoction permet de recueillir le plus de principes actifs et atténue ou annule l'effet toxique de certaines drogues. Quant à la macération et l'infusion, elles permettent de 
préserver l'intégrité des principes actifs (Salhi et al., 2010). Ces préparations sont presque toutes administrées en boisson (voie orale) et quelques fois en purge (voie rectale). Cela peut s'expliquer par la simplicité de ce mode d'administration mais aussi par le fait que les drogues étant sous forme brute, la voie orale s'avère la moins dangereuse, l'absorption des principes actifs se faisant au niveau de l'intestin grêle.

Ce travail a montré que les recettes plurispécifiques $(77,88)$ sont majoritairement représentées par rapport aux recettes monospécifiques $(22,12 \%)$. Ces résultats confirment ceux de Kipré et al. (2017), réalisés sur les plantes antipaludiques, dans le département d'Agboville. Ces auteurs ont enregistré $62 \%$ de recettes plurispécifiques. Les associations de plantes permettent de renforcer l'activité antiplasmodiale. Par exemple, l'association d'une fraction acétique de Dalium dinklagei et d'une fraction butanolique de Cnestis ferruginea a montré une activité antiplasmodiale plus importante par effet de synergie (Tuo, 2015). En outre, elle permet de créer une dynamisation des effets curatifs des divers constituants afin d'aboutir à une synergie thérapeutique combattant à la fois le parasite responsable de la maladie et agissant aussi contre certains symptômes associés tels que la fatigue générale, le manque d'appétit et la fièvre (Betti, 2002 ; Bla et al., 2015). Contrairement aux résultats obtenus dans ce travail, ceux obtenus par Ouattara (2006) à Divo et par Bla et al. (2015) à Toumodi indiquent que les recettes monospécifiques sont majoritairement représentées avec respectivement des proportions de $87,50 \%$ et $69,23 \%$. Ces auteurs soutiennent que cette prépondérance est à l'avantage des patients car les associations de plantes, mal assorties, sont parfois dangereuses et que l'usage des mixtures est la cause d'environ $30 \%$ des accidents mortels en Afrique.

\section{Conclusion}

Cette étude a montré une diversité de 54 espèces de plantes utilisées par les tradithérapeutes et les herboristes dans la ville
d'Abidjan pour traiter le paludisme. Les feuilles et la décoction sont respectivement les organes végétaux et le mode de préparation médicamenteuse les plus utilisés. Les remèdes sont administrés surtout par voie orale, mais quelques fois par voie rectale. Des études phytochimiques et pharmacologiques sont en cours sur certaines de ces plantes en vue de contribuer à valider leur utilisation traditionnelle et de trouver de nouvelles plantes à potentiel antipaludique.

\section{CONFLIT D'INTÉRÊTS}

Les auteurs déclarent qu'il n'existe aucun conflit d'intérêts.

\section{CONTRIBUTIONS DES AUTEURS}

YS a élaboré le questionnaire, conduit les enquêtes sur le terrain, pris part à la rédaction et a approuvé la version finale du manuscrit ; DKS a participé à l'élaboration du questionnaire, à la rédaction et à la correction et a approuvé la version finale du manuscrit ; KO a participé à l'élaboration du questionnaire et aux enquêtes sur le terrain ; MWK a développé le concept de l'étude, guidé l'élaboration du questionnaire, supervisé les enquêtes sur le terrain, participé à la rédaction et à la correction et a approuvé la version finale du manuscrit.

\section{REMERCIEMENTS}

Les auteurs tiennent à remercier tous les tradithérapeutes et les herboristes pour leur disponibilité et leur franche collaboration a cette étude. Ils remercient également, Docteur Ehoulé KROA, Directeur du Programme national de promotion de la médecine traditionnelle en Côte d'Ivoire, pour avoir facilité l'accès aux tradithérapeutes et herboristes. Ils remercient enfin, Monsieur DOUGOUNE Saturnin et Monsieur ASSI Yapo Jean, techniciens en botanique respectivement au CSRS et au CNF (UFHB), pour leur contribution à l'identification des plantes recensées.

\section{REFERENCES}

Adomou AC, Yedomonhan H, Djossa B, Legba SI, Oumorou M, Akoegninou A. 
2012. Etude Ethnobotanique des plantes médicinales vendues dans le marché d'Abomey-Calavi au Bénin. Int. J. Biol. Chem. Sci., 6(2): 745-772. DOI: http://dx.doi.org/10.4314/ijbcs.v6i2.18

Ambé ASA. 2006. Inventaire de la flore et étude structurale de la végétation d'une parcelle de la forêt classée de Monogaga sud (San-Pedro, Côte d'Ivoire). Mémoire de D.E.A, Université de CocodyAbidjan, 78p.

Ambé ASA. 2016. Plantes médicinales utilisées dans le traitement traditionnel de la diarrhée, à Abidjan (Côte d'Ivoire) étude ethnobotanique, évaluation des activités antimicrobiennes et antioxydantes de deux espèces. Thèse de Doctorat Unique, Université Félix Houphouët Boigny, Abidjan, 164p.

Benlamdini N, Mohamed E, Atmane R, Lahcen Z. 2014. Etude floristique et ethnobotanique de la flore médicinale du Haut Atlas oriental (Haute Moulouya), Maroc. J. Appl. Biosci., 78: 6771-6787. DOI: http://dx.doi.org/10.4314/jab.v78i1.17

Bétti JL. 2002. Medicinal plants sold in Yaoundé markets, Cameroon. Afr. St. Mono., 23(3): 47-64. DOI: http://dx.doi.org/10.14989/68215

Bla KB, Trebissou JND, Bidie A. 2015. Étude ethnopharmacologique des plantes antipaludiques utilisées chez les BaouléN'Gban de Toumodi dans le Centre de la Côte d'Ivoire. J. Appl. Biosci., 85: 77757783.

DOI:

http://dx.doi.org/10.4314/jab.v85i1.4

Bureau National d'Etude Technique et de Développement (BNETD). Les cahiers d'information du CCT N${ }^{\circ}, 1997$.

Dasylva B. 2001. Contribution à l'étude de l'herboristerie traditionnelle sénégalaise: Inventaire des plantes médicinales vendues dans les marchés de Dakar et contrôle de qualité sur 170 échantillons. Thèse doctorat pharmacie, Université Cheikh Anta Diop, Dakar, 144p.

Dénou A, Koudouvo K, Togola A, Haïdara M, Dembélé SM, Ballo FN, Sanogo R,
Diallo D, Gbéassor M. 2017. Savoir traditionnel sur les plantes antipaludiques à propriétés analgésiques, utilisées dans le district de Bamako (Mali). J. Appl. Biosci., 112 :1098510995.

DOI : https://dx.doi.org/10.4314/jab.v112i1.3

Hermans $M$, Akoègninou $\mathrm{A}$, van der Maesen LJG. 2004. Medicinal plants used to treat malaria in southern Benin. Economic Botany, 58: S239-S252. DOI: http://dx.doi.org/10.16663/0013-0001 (2004)50[S2: MPUTTM] 2.0.CO; 2

Ilumbe GB, Van-Damme P, Lukoki FL, Joris V, Vissier M, Lejoly J. 2004. Contribution à l'étude des plantes médicinales dans le traitement des hémorroïdes par les pygmées Twa et leur voisin Oto de Bikoro, en RDC. Congo Sciences, 2(1): 46-54. URL: http://hdl.handle.net/1854/LU-4358485

Kipré GR, Offoumou MBR, Silue KD, Bouabre GM, Zirihi Guédé N, Djaman AJ.2017. Enquête ethnopharmacologique des plantes antipaludiques dans le département d'Agboville, sud-est de la Cote d'Ivoire. J. Appl. Biosci., 109: 10618-10629.

DOI: http://dx.doi.org/10.4314/jab.v109i1.6

Koné MW. 2005. Potentiel des plantes médicinales de Côte-d'Ivoire dans le contrôle des hoemonchoses chez les ovins. Thèse de Doctorat, Université de Cocody, Abidjan 202p.

Mangambu MJD., Mushagalusa KF, Kadima NJ, 2014. Contribution à l'étude phytochimique de quelques plantes médicinales antidiabétiques de la ville de Bukavu et ses environs (Sud-Kivu, R.D. Congo). J. Appl. Biosci., 75 : 6211-6220. DOI: http://dx.doi.org/10.4314/jab.v75i1.7

N'Guessan K. 2008. Plantes médicinales et pratiques médicinales traditionnelles chez les peuples Abbey et Krobou du département d'Agboville (Côte d'Ivoire). Thèse de Doctorat d'Etat, Université de Cocody, Abidjan, 335 p.

OMS. 2004. Atelier sur l'institutionnalisation de la Médecine Traditionnelle dans les 
systèmes de santé, Cotonou, Bénin, du 13 au 15 septembre 2004. OMS.

OMS. 2011. Rapport 2011 sur le paludisme dans le monde. Résumé et points essentiels. 3p. OMS.

OMS. 2016. World Malaria Report 2016. 186p. OMS.

Ouattara D. 2006. Contribution à l'inventaire des plantes médicinales significatives utilisées dans la région de Divo (sud forestier de la Côte-d'Ivoire) et à la diagnose du poivrier de Guinée : Xylopia aethiopica (Dunal) A. Rich. (Annonaceae). Thèse de Doctorat, Université de Cocody, Abidjan, 184 p.

Pradines B, Dormoia J, Briolanta S, Bogreaua H, Rogiera C. 2010. La résistance aux antipaludiques, Rev. Francoph. Lab., 422: 51-61. DOI: $10.1016 / \mathrm{S} 1773-035 \mathrm{X}$ (10)70510-4

Salhi S, Fadli M, Zidane L, Douira A, 2010. Études floristique et ethnobotanique des plantes médicinales de la ville de Kénitra (Maroc). Lazaroa, 31: 133-146. DOI: https://doi.org/10.5209/rev_LAZA.2010. v31.9

Sanon S, Ollivier E, Azas N, Mahiou V, Gasquet M, Ouattara CT, Nebie I, Traore AS, Esposito F, Balansard G, TimonDavid P, Fumoux F. 2002. Ethnobotanical survey and in vitro antiplasmodial activity of plantsused in the traditional medicine in Burkina Faso. J. Ethnopharmacol., 86: 143-147. DOI: https://doi.org/10.1016/S03788741(02)00381-1
Société d'Exploitation et de développement Aéroportuaire Aéronautique et Météorologiques (SODEXAM). 2015. Données météorologiques de 2004 à 2014 de la Ville d'Abidjan. Direction de la Météorologie Nationale, Port-BouëtAbidjan (Côte d'Ivoire).

Togola A, Diallo D, Dembélé S, Barsett H, Paulsen SB. 2005. Ethnopharmacological survey of different uses of seven medicinal plants from Mali (West Africa) in the regions Diola, Kolokani and Siby. J Ethnobiol Ethnomed, 1: $7 . \quad$ DOI: https://doi.org/10.1186/1746-4269-1-7

Tuo K. 2015. Criblage phytochimique, activité antioxydante et antiplasmodiale in vitro de cinq plantes utilisées traditionnellement en Côte d'Ivoire contre le paludisme. Thèse de Doctorat Unique, Université Félix Houphouët Boigny, Abidjan, 200p.

Yapi AB. 2013. Inventaire des plantes médicinales de la famille des Asteraceae des marchés de la commune d'Abobo (Abidjan, Côte d'Ivoire). Mémoire de Master II de botanique, Université Félix Houphouët-Boigny, Abidjan, 50p.

Zirihi GN. 2006. Etude botanique, pharmacologique et phytochimique de quelques plantes antipaludiques et/ou immunogènes utilisées chez les Bétés du département d'Issia dans l'Ouest de la Côte d'Ivoire. Thèse de Doctorat d'Etat, Université de Cocody, Abidjan, 126 p. (BNETD, 1997). 